\title{
Editorial
}

\section{A Note of Appreciation}

This journal's impact factor in 2006 was 4.33. This confirms Psychotherapy and Psychosomatics as a European leading journal in both the SCI Psychiatry (14th) and Psychology (4th) rankings.

In psychology it is the top journal among those which publish original investigations. It has become the home of unconventional thinking and innovative ideas in behavioral and medical sciences. This target would not have been achieved without the help of the external referees, who have supported the editorial board in reviewing the manuscripts submitted to Psychotherapy and Psychosomatics.

Both external referees and editorial board members have disclosed potential conflicts of interest. The editorin-chief has no conflict of interest to declare for 2007.
Abbate-Daga G. (Turin, Italy) Andreoli A. (Geneva, Switzerland) Barbosa A. (Lisboa, Portugal) Barbui C. (Verona, Italy) Battaglia M. (Milan, Italy) Baune B. (Townsville, Australia) Belaise C. (Bologna, Italy) Benazzi F. (Forlì, Italy) Benninghoven D. (Lübeck, Germany) Bernardi M. (Bologna, Italy) Berrocal C. (Malaga, Spain) Bockting C.L.H. (Groningen, The Netherlands) Bohus M. (Mannheim, Germany) Bouvard M. (Chambery, France) Bravi A. (Bologna, Italy) Büchi S. (Zurich, Switzerland) Carta M. (Cagliari, Italy) Chiesa M. (Richmond, UK) Corazziari E. (Rome, Italy) Costantini A. (Rome, Italy) Davidson K. (New York, N.Y., USA) De Figuereido J.C. (Cheshire, UK) de Fruyt J. (Brugge, Belgium) delle Fave A. (Milan, Italy) Facchinetti F. (Modena, Italy) Faravelli C. (Florence, Italy) Fassino S. (Turin, Italy) Fink P. (Aarhus, Denmark) Forstmeier S. ( Zurich, Switzerland) Fullana M.A. (London, UK) Grabe H.J. (Greifswald/Stralsund, Germany)
Grigioni F. (Bologna, Italy)

Grossi G. (Stockholm, Sweden)

Guidi J. (Bologna, Italy)

Hengeveld M. (Rotterdam, The Netherlands)

Hoglend P. (Oslo, Norway)

Holmes-Rovner M. (East Lancing, Mich., USA)

Honkalampi K. (Kuopio, Finland)

Jarrett R.B. (Dallas, Tex., USA)

Juckel G. (Bochum, Germany)

Kocalevent R.D. (Berlin, Germany)

Kudielka B.M. (Trier, Germany)

Leombruni P. (Turin, Italy)

Levenstein S. (Rome, Italy)

Linley A. (Leicester, UK)

Lumley M.A. (Detroit, Mich., USA)

Maina G. (Torino, Italy)

Marissen M.A.E. (The Hague, The Netherlands)

Mataix-Cols D. (London, UK)

Metternich B. (Freiburg, Germany)

Moncrieff J. (London, UK)

Moritz S. (Aachen, Germany)

Morosini P.L. (Rome, Italy)

Mota Cardoso R. (Porto, Portugal)

Natale V. (Bologna, Italy)

Nielson K. (Milwaukee, Wisc., USA)

Ogrodniczuk J.S. (Vancouver, Canada)

Orth-Gomer K. (Stockholm, Sweden)

Ottolini F. (Modena, Italy)

Patten S.B. (Calgary, Canada)

Peretti C. (Paris, France)
Picardi A. (Rome, Italy)

Piccirillo G. (Rome, Italy)

Radanov B. (Zurich, Switzerland)

Rafanelli C. (Bologna, Italy)

Rapin I. (Bronx, N.Y., USA)

Rigatelli M. (Modena, Italy)

Rihmer Z. (Budapest, Hungary)

Romeis C. (Geneva, Switzerland)

Roncuzzi R. (Bologna, Italy)

Rufer M. (Zurich, Switzerland)

Salmaso L. (Padova, Italy)

Sanavio E. (Padua, Italy)

Schedlowski M. (Essen, Germany)

Schneider G. (Münster, Germany)

Semprini F. (Bologna, Italy)

Sharpe L. (Sydney, Australia)

Simons M. (Kaachen, Germany)

Sirri L. (Bologna, Italy)

Sobrinho L. (Lisboa, Portugal)

Spinhoven P. (Leiden, The Netherlands)

Stangier U. (Jena, Germany)

Starcevic V. (Sydney, Australia)

Stuart S. (Iowa City, Iowa, USA)

Tarrier N. (Manchester, UK)

Tossani E. (Bologna, Italy)

Van Emmerik A.A.P. (Amsterdam,

The Netherlands)

Vanheule S. (Gent, Belgium)

Van Houdenhove B. (Leuven, Belgium)

Wiltink J. (Mainz, Germany)

Zachariae R. (Aarhus, Denmark)

Zimmerman C. (Verona, Italy)

\section{KARGER}

Fax +41613061234

E-Mail karger@karger.ch

www.karger.com
(C) 2008 S. Karger AG, Basel

0033-3190/08/0771-0003\$24.50/0

Accessible online at:

www.karger.com/pps 